\title{
Improved Phase I Control Charts for Monitoring Times Between Events
}

\author{
N. Kumar ${ }^{* \dagger}$ and S. Chakraborti $\ddagger$
}

\begin{abstract}
In many situations the times between certain events are observed and monitored instead of the number of events themselves, particularly when the events occur rarely. In this case it is common to assume that the times between events follow an exponential distribution. Control charts are one of the main tools of statistical process control and monitoring. Control charts are used in Phase I to assist operating personnel in bringing the process into a state of statistical control. In this paper, Phase I control charts are considered for the observations from an exponential distribution with an unknown mean. A simulation study is carried out to compare the in-control (IC) robustness and out-of-control (OOC) performance of the proposed chart. It is seen that the proposed charts are considerably more IC robust than two competing charts and have comparable OOC properties.
\end{abstract}

keywords: Phase I and Phase II control charts; Exponential distribution; In-control robustness; TBE; Performance; Dixon's statistic.

\section{Introduction}

A control chart is a very useful statistical tool used to distinguish between common and special causes of variation. In the literature, two phases of control charting practice have been discussed. In Phase I, a set of process data is gathered and

${ }^{*}$ Department of Statistics, University of Pretoria, Hatfield, South Africa

${ }^{\dagger}$ Correspondence to: Department of Statistics, University of Pretoria, Hatfield, South Africa, Email: nirpeksh@gmail.com, Mob.:+27-740717125

${ }^{\ddagger}$ Department of Information Systems, Statistics and Management Science, University of Alabama, Tuscaloosa, AL 35487, USA 
analyzed all at once in a retrospective analysis, constructing trial control limits to determine if the process has been in control over the period of time where the data were collected, and to see if reliable control limits can be established to monitor the future production process. Control charts are used primarily in Phase I to assist operating personnel in bringing the process into a state of statistical control. In Phase II, we use the control chart to monitor the process by comparing the sample statistic for each successive sample as it is drawn from the process to the control limits. The Phase I or the retrospective phase is an important component of an overall control charting and monitoring regime where the objectives are somewhat different from the Phase II or the monitoring phase. The reader is referred to Chakraborti et al. ${ }^{1}$ for an overview of Phase I control charts.

In many situations the time between certain events are observed and monitored instead of the number of event occurrences, particularly when the events occur rarely. In this case it is common to assume that the times between events (TBE) follow an exponential distribution. Montegomery ${ }^{2}$ recommends that the times between failures be monitored with regard to the quality of the process. Several TBE control charts have been proposed in the literature ${ }^{3-5}$. Liu et al. ${ }^{6}$ compare some of these charts including the CUSUM and EWMA charts. Some authors suggest applying the regular Shewhart charts after a transformation of the data ${ }^{7-9}$. Jones and Champ ${ }^{10}$ emphasize the early stage process improvement activities when failures occur according a homogeneous Poisson process. Jones and Champ ${ }^{10}$ They also addressed the seriousness of the problem when the Phase I control chart is used to control the individual false alarm rate and suggested that the Phase I control charts should be designed to control the overall false alarm rate (probability) and not the individual false alarm rate.

Robustness is an important desirable property of a control chart so that the IC chart performance is stable. If a control chart is not IC robust, its performance properties become somewhat pointless. Robustness of control charts has been discussed by many authors ${ }^{11-13}$. Dovoedo and Chakraborti ${ }^{13}$ examined the robustness Jones and Champ ${ }^{10}$ charts and proposed new Phase I control charts based on a modified box-plot for individual observations from an exponential distribution with an unknown mean, so that the overall false alarm rate is controlled at a given nominal value. In this paper, we improve on these charts further and consider new Phase I control charts for observations from an exponential distribution with an unknown mean.

The key idea is that since the proposed charts are constructed from the median of the data, the resulting control limits are expected to be more IC robust than those proposed by Jones and Champ ${ }^{10}$, which are based on the mean, in the presence of 
one or more OOC observations.

The paper is structured as follows: In Section 2, the control limits for the onesided control chart are proposed. The control limits for two-sided control chart are developed in Section 3. A simulation study is carried out to compare the in-control robustness of the proposed procedure with the Jones and Champ ${ }^{10}$ and Dovoedo and Chakraborti ${ }^{13}$ procedures in Section 4 and is compared the OOC performance in Section 5. In section 6, a numerical example is given for illustration and the concluding remarks are made in Section 7.

\section{The One-sided control chart}

Suppose that the events in a process occur according to a homogeneous Poisson process with a constant failure rate. Under this assumption, the times between two consecutive failures are independent and follow an exponential distribution. Further suppose that there are $n$ random times between failures, denoted by $X_{i}, i=1, \ldots, n$. Assume that these random variables $X_{1}, \ldots, X_{n}$ are independent and follows an exponential distribution with an unknown mean $\mu_{1}, \ldots, \mu_{n}$ respectively. The process is IC at time $i$ when $\mu_{i}=\mu$ (unknown). Let $\bar{X}=(1 / n) \sum X_{i}$ denote the sample average and let $\alpha$ denote the overall false alarm rate. Jones and Champ ${ }^{10}$ proposed the following one-sided control chart to maintain the overall false alarm rate at a nominal level $\alpha_{0}$.

$$
\begin{aligned}
\mathrm{LCL} & =\left[1-\left(1-\alpha_{0}\right)^{1 /(n-1)}\right] \bar{X} \\
\mathrm{CL} & =\bar{X}
\end{aligned}
$$

Let $X_{(1)}, \ldots, X_{(n)}$ be the ordered observations and let $X_{(l)}, X_{(m)}, X_{(u)}$ denote the first, the second and the third quartile, respectively. Dovoedo and Chakraborti ${ }^{14}$ use the following definition for the indices $l, m$, and $u: m=\operatorname{ceil}(m / 2) ; l=f l o o r(m / 4)+1$ if $\bmod (m, 4) \neq 0$ and $l=f l o o r(m / 4)$ otherwise, where ceil $(a)$ denotes the smallest integer greater than or equal to $a$, floor $(a)$ denotes the largest integer less than or equal to $a$, and $\bmod (a, b)$ denotes the remainder in the division of an integer $a$ by an integer $b ; u=m-l+1$.

Motivated by a boxplot, Dovoedo and Chakraborti ${ }^{13}$ proposed the following lower control limit and the center line for a one-sided chart for the same problem.

$$
\begin{aligned}
\mathrm{LCL} & =X_{(m)}-k_{l}\left(X_{(m)}-X_{(l)}\right) \\
\mathrm{CL} & =X_{(m)}
\end{aligned}
$$

The constant $k_{l}$ is called a fence constant, which is determined to control the overall false alarm rate at some nominal level $\alpha_{0}$. Thus $k_{l}$ is found such that 


$$
\begin{aligned}
\alpha_{0} & =\operatorname{Pr}\left[X_{(1)}<X_{(m)}-k_{l}\left(X_{(m)}-X_{(l)}\right) \mid I C\right] \\
& =\int_{0}^{\infty} \int_{0}^{\infty} I_{G_{l}\left(y_{l}\right)}(\alpha=1, \beta=l-1) f_{Z_{(l)}, Z_{(m)}}\left(z_{(l)}, z_{(m)}\right) d z_{(m)} d z_{(l)}
\end{aligned}
$$

where $y_{(l)}=z_{(m)}-k_{l}\left(X_{(m)}-X_{(l)}\right), I_{G_{l}\left(y_{l}\right)}(\alpha=1, \beta=l-1)$ denotes the incomplete Beta function evaluated at $G_{l}\left(y_{(l)}\right)=F\left(y_{(l)}\right) / F\left(z_{(l)}\right)$ and $z_{(i)}$ denotes the value of $Z_{(i)}$, the $i$ th order statistic of a random sample of size $n$ from the distribution of the standardized exponential random variable $Z=X / \mu$.

Dovoedo and Chakraborti ${ }^{13}$ suggested the lower control limit based on the spacing between the median and the first quartile, $X_{(l)}$ and $X_{(m)}$. As the observation $X_{(l+1)}$ is expected to be mostly affected directly by its predecessor $X_{(l)}$, we consider a new lower control limit for the one-sided chart, based on the the spacing between these two order statistics, as

$$
\begin{aligned}
\mathrm{LCL} & =X_{(m)}-k_{1}\left(X_{(l+1)}-X_{(l)}\right) \\
\mathrm{CL} & =X_{(m)}
\end{aligned}
$$

For a given nominal value $\alpha_{0}$ the new fence constant $k_{1}$ is obtained from the following equation, which is the overall false alarm rate, that is the probability of the event that at least one observation falls below the lower control limit when the process is IC.

$$
\alpha_{0}=\operatorname{Pr}\left[X_{(1)}<X_{(m)}-k_{1}\left(X_{(l+1)}-X_{(l)}\right) \mid I C\right] .
$$

Note that the above equation may be re-written as

$$
\alpha_{0}=\operatorname{Pr}\left[\frac{X_{(l+1)}-X_{(l)}}{X_{(m)}-X_{(1)}}<1 / k_{1} \mid I C\right] .
$$

Now denoting

$$
T_{1}=\frac{X_{(l+1)}-X_{(l)}}{X_{(m)}-X_{(1)}}
$$

The fence constant $k_{1}$ for the new procedure can be obtained as

$$
\alpha_{0}=\operatorname{Pr}\left[T_{1}<1 / k_{1} \mid I C\right] .
$$

It turns out that the statistic $T_{1}$ is a particular case of Dixon's (Dixon ${ }^{15}$ ) statistic. Likes ${ }^{16}$ obtained its distribution for testing outliers in an exponential sample, which 
is given in the appendix. Thus, the IC distribution of the statistic $T_{1}$ can be simply obtained by substituting suitable quantities $(s=l+1, r=l, q=m, p=1)$ in the statistic (A.1)and its distribution (A.2). This is given by

$$
\begin{aligned}
1 & -F_{T_{1}}(t) \\
=\frac{(n-1) !}{(n-m) !}(1-t) & {\left[\sum_{i=1}^{m-l-1}\left[\frac{(-1)^{i+1}(m-l-i)[(n-l) t+(n-m+i)(1-t)]^{-1}}{(i-1) !(m-i-1) !(n-l)}\right]\right.} \\
& \left.+\sum_{j=1}^{l-1}\left[\frac{(-1)^{m-l+j} j[(n-l) t+(n-l+j)(1-t)]^{-1}}{(l-j-1) !(m-l+j-1) !(n-l)}\right]\right]
\end{aligned}
$$

Since equation (6) may be written as

$$
\alpha_{0}=F_{T_{1}}\left(1 / k_{1}\right)=F_{T_{1}}\left(t_{1}\right)
$$

with $t_{1}=1 / k_{1}$. The new fence constant can be obtained using (7).

Thus for a fixed nominal value $\alpha_{0}$, equation (8) can be solved numerically (using, for example, the solve function in MATLAB), where $F_{T_{1}}\left(t_{1}\right)$ is given by $(7)$. Once the fence constant $k_{1}$ is found, the lower control limit of the proposed one-sided control chart can be constructed by using equation (4). The values of the fence constant $k_{1}$ for some selected values of $n$ and some typical values of the overall false alarm rate $\alpha_{0}$ are presented in Table 1.

\section{The two-sided control chart}

Jones and Champ ${ }^{10}$ give the approximate lower and upper control limits to maintain the overall false alarm rate at the nominal level $\alpha_{0}$ as follows:

$$
\begin{aligned}
\mathrm{LCL} & =\frac{n \bar{X}}{1+(n-1) F_{2(n-1), 2}\left(1-\alpha_{0} / n+\tau\right)} \\
\mathrm{CL} & =\bar{X} \\
\mathrm{UCL} & =\frac{n \bar{X}}{1+(n-1) F_{2(n-1), 2}(\tau)}
\end{aligned}
$$

where the $F$-distribution has numerator and denominator degrees of freedom $2(n-1)$ and 2 ; and $\tau$ satisfies $0<\tau<\alpha_{0} / n$. 
For the same problem, Dovoedo and Chakraborti ${ }^{13}$ proposed the two-sided control limits, for a given nominal value $\alpha_{0}$,

$$
\begin{aligned}
\mathrm{LCL} & =X_{(m)}-k_{l}\left(X_{(m)}-X_{(l)}\right) \\
\mathrm{CL} & =X_{(m)} \\
\mathrm{UCL} & =X_{(m)}+k_{u}\left(X_{(u)}-X_{(m)}\right)
\end{aligned}
$$

We propose new control limits for two-sided control chart based on the spacing $X_{(u)}-X_{(u-1)}$ and $X_{(l+1)}-X_{(l)}$ as the observation $X_{(u)}$ is most affected by $X_{(u-1)}$ and $X_{(l+1)}$ by $X_{(l)}$. Hence the proposed two-sided control chart is given by

$$
\begin{aligned}
\mathrm{LCL} & =X_{(m)}-k_{1}\left(X_{(l+1)}-X_{(l)}\right) \\
\mathrm{CL} & =X_{(m)} \\
\mathrm{UCL} & =X_{(m)}+k_{2}\left(X_{(u)}-X_{(u-1)}\right)
\end{aligned}
$$

The fence constants $k_{1}$ and $k_{2}$ may be obtained using the following statistics

$$
\begin{aligned}
& T_{1}=\frac{X_{(l+1)}-X_{(l)}}{X_{(m)}-X_{(1)}} \\
& T_{2}=\frac{X_{(u)}-X_{(u-1)}}{X_{(n)}-X_{(m)}}
\end{aligned}
$$

The IC distribution of $T_{1}$ is given by (7). The statistic $T_{2}$ and its IC distribution can be obtained by replacing $s=u, r=u-1, q=m$ and $p=m$ in equations (A.1) and (A.2). This is given by

$$
\begin{aligned}
& 1- F_{T_{2}}(t) \\
&=(n-m) !(1-t)\left[\sum_{i=1}^{n-u}\left[\frac{(-1)^{i+1}(n-u-i+1)[(n-u+1) t+i(1-t)]^{-1}}{(i-1) !(n-m-i) !(n-u+1)}\right]\right. \\
&\left.\quad+\sum_{j=1}^{u-m-1}\left[\frac{(-1)^{n-u+j+1} j[(n-u+1) t+(n-u+j+1)(1-t)]^{-1}}{(u-m-j-1) !(n-u+j) !(n-u+1)}\right]\right]
\end{aligned}
$$

Thus the overall false alarm rate is given by

$$
\alpha=\operatorname{Pr}\left[X_{(1)}<X_{(m)}-k_{1}\left(X_{(l+1)}-X_{(l)}\right) \cup X_{(n)}>X_{(m)}+k_{2}\left(X_{(u)}-X_{(u-1)}\right) \mid I C\right]
$$

Hence for a nominal value $\alpha_{0}$ of the overall false alarm rate, the fence constants $k_{1}$ and $k_{2}$ are obtained such that

$$
\alpha_{0}=\operatorname{Pr}\left[X_{(1)}<X_{(m)}-k_{1}\left(X_{(l+1)}-X_{(l)}\right) \cup X_{(n)}>X_{(m)}+k_{2}\left(X_{(u)}-X_{(u-1)}\right) \mid I C\right]
$$


that is

$$
\alpha_{0}=\operatorname{Pr}\left[T_{1}<1 / k_{1} \cup T_{2}<1 / k_{2} \mid I C\right] .
$$

Using the property of the exponential distribution, it can be seen that the events $\operatorname{Pr}\left[T_{1}<1 / k_{1}\right]$ and $\operatorname{Pr}\left[T_{2}<1 / k_{2}\right]$ are independent but are not disjoint. Hence (14) may be written as

$$
\begin{aligned}
\alpha_{0} & =\operatorname{Pr}\left[T_{1}<1 / k_{1} \mid I C\right]+\operatorname{Pr}\left[T_{2}<1 / k_{2} \mid I C\right]-\operatorname{Pr}\left[T_{1}<1 / k_{1} \cap T_{2}<1 / k_{2} \mid I C\right] \\
& =\operatorname{Pr}\left[T_{1}<1 / k_{1} \mid I C\right]+\operatorname{Pr}\left[T_{2}<1 / k_{2} \mid I C\right]-\operatorname{Pr}\left[T_{1}<1 / k_{1} \mid I C\right] . \operatorname{Pr}\left[T_{2}<1 / k_{2} \mid I C\right]
\end{aligned}
$$

Thus, one way to find the constants $k_{1}$ and $k_{2}$ from (15) is to make the term $\operatorname{Pr}\left[T_{1}<1 / k_{1} \mid I C\right]$ in equation (15) equal to $\alpha_{0} /\left(2-\alpha_{0}\right)$ and the term $\operatorname{Pr}\left[T_{2}<1 / k_{2} \mid I C\right]$ equal to $\alpha_{0} / 2$. Following this line of argument, the charting constants $k_{1}$ and $k_{2}$ are calculated and provided in Table 2 for some selected values of $n$ and the overall nominal false alarm rate $\alpha_{0}$.

\section{Comparison of IC robustness}

Here, we first investigate the IC robustness of the one-sided control charts to the assumption of the underlying exponential distribution via simulation. Similar comparisons are then done for the two-sided charts. The IC robustness is an important attribute of a control chart and should be investigated thoroughly since in practice the underlying distribution may not be exactly exponential. The more robust the control chart, the more confidence the user has on the advertised false alarm rate associated with that control chart. Without the assurance of a robust false alarm rate, the performance of a control chart in detecting changes by a OOC signal becomes somewhat meaningless.

The simulation study is modeled after Dovoedo and Chakraborti ${ }^{13}$. For this, we consider two slightly more and two slightly less skewed distributions than the exponential distribution. The two more skewed distributions are the $\operatorname{Gamma}(1.1,1)$ and the $\operatorname{Gamma}(1.2,1)$ and the two less skewed distributions are the $\operatorname{Gamma}(0.8,1)$ and the Gamma(0.9,1), respectively. The results in this section are based on 100,000 simulations with a sample of $n=20$ observations. The results for the one-sided control charts are reported in the Table 3.

From Table 3, it is seen that in almost all cases, the empirical overall false alarm rates for the Jones and Champ ${ }^{10}$ chart deviate most significantly from the nominal overall false alarm rate $\alpha_{0}$, whereas those for the proposed chart deviate the 
least. In other words, in all of the 16 cases considered, the proposed procedure is most IC robust in terms of the overall false alarm rate. For example, for the Gamma $(0.8,1)$ distribution with $\alpha_{0}=0.2$, the absolute deviation of the overall false alarm rate is $96.0 \%$ for the Jones and Champ ${ }^{10}$ chart, it is $29.8 \%$ for the Dovoedo and Chakraborti ${ }^{13}$ chart, but it is only $0.9 \%$ for the proposed chart. Moreover, the variation among the empirical overall false alarm rates is also seen to be negligible for the proposed control chart for any given nominal probability $\alpha_{0}$. Thus the proposed one-sided chart is far more IC robust than both of its competitors, the Jones and Champ ${ }^{10}$ chart and the Dovoedo and Chakraborti ${ }^{13}$ chart.

A similar simulation study was carried out for two-sided control charts and the results are presented in the Table 4 . To compute the control limits for Jones and Champ ${ }^{10}$ charts, $\tau$ was taken to be equal to $\alpha_{0} / 2 n$, the mid-point between 0 and $\alpha_{0} / n$ in equation (9).

From Table 4, we reach the same conclusion as for Table 3. The empirical false alarm rates for the Jones and Champ ${ }^{10}$ chart deviate most significantly from the desired overall nominal false alarm rate, whereas the proposed procedure is seen to be the most robust. In fact in all the 16 cases, the proposed procedure turns out to be the most robust. For example, for the Gamma(1.2,1) distribution, using $\alpha_{0}=0.2$, the deviation is $53.1 \%$ for the Jones and Champ ${ }^{10}$ chart, $10.7 \%$ for Dovoedo and Chakraborti ${ }^{13}$ whereas it is only $1.6 \%$ for the proposed chart. This is a significant improvement.

A simulation study was also done to study the impact of $n$. It is seen that as the sample size increases the Jones and Champ ${ }^{10}$, Dovoedo and Chakraborti ${ }^{13}$ procedures both become less IC robust than the proposed chart. However, the proposed chart remains almost robust for all $n$. As the nominal value $\alpha_{0}$ increases, the charts other than the proposed chart deviate more significantly whereas the proposed chart shows the same robustness in terms of overall nominal false alarm rate $\alpha_{0}$.

Next we examine the OOC performance of the charts.

\section{The OOC performance}

To compare the out-of-control performance of the proposed charting procedure with the other two, we follow the same set up as in Dovoedo and Chakraborti ${ }^{13}$. We use the shift values for $\delta$ : $-0.05 ;-0.1 ;-0.25$; and -0.5 .

Tables 5 and 6 show the performance of the one-sided and two-sided control charts, respectively, in terms of the observed proportions of out-of-control signals. All these results are based on 100,000 simulations with $n=20$. The $95 \%$ margin of error for reported results in Tables 5 and 6 is approximately 0.00295 . 
It is seen that the proposed charts are comparably effective in detecting an OOC situation. For example for a nominal value $\alpha_{0}=0.1$, with 5 out of the 20 observations replaced by OOC observations from $\operatorname{Expo}(0.5)(\delta=-0.5)$, the proportion of cases with at least one signal is $0.10116=10.116 \%$ for the proposed procedure for the one-sided Phase I control chart, while it is $0.09115=9.115 \%$ for Dovoedo and Chakraborti ${ }^{13}$ and $0.10156=10.156 \%$ for Jones and Champ ${ }^{10}$ chart. It may be noted that none of these charts seems entirely superior or satisfactory in respect of OOC performance. However, the key for a Phase I is a robust IC performance.

Next we illustrate the proposed control chart with an example.

\section{An Illustration}

Table 7 shows a set of 30 failure time data generated from a Poisson distribution with a mean of 0.1 . For these data $n=30, l=8, m=15$ and $u=23$. We monitor these data with the proposed Phase I chart.

The center line for the proposed two-sided control chart is $\mathrm{CL}=X_{(15)}=6.91$ and the lower and upper control limits are given by:

$$
\mathrm{LCL}=-53.9213 \quad \text { and } \quad \mathrm{UCL}=47.2320
$$

Since LCL $<0$ we fix the lower control limit as LCL $=0$. It can be seen from Figure 1 that the 11th observation 52.32 plots outside the UCL which indicates an OOC situation, that needs further invetigation. Note that for these data, neither the Dovoedo and Chakraborti ${ }^{13}$ nor the Jones and Champ ${ }^{10}$ control chart indicates any OOC situation.

\section{Concluding remarks}

In this paper Phase I control charts are considered for observations from an exponential distribution with an unknown mean. The proposed charts are based on the median and hence hold the IC robustness property well. In fact the proposed charts are shown to be more IC robust than both the Jones and Champ ${ }^{10}$ and the Dovoedo and Chakraborti ${ }^{13}$ charts currently available in the literature. Further work is necessary on the out-of-control performance of these charts. 


\section{Acknowledgements}

The first author's post doctoral fellowship is supported by a South African Research Chairs Initiative (SARChI) award to the second author at the University of Pretoria, in South Africa. Partial support is also provided by the Department of Statistics, University of Pretoria.

\section{A The distribution of Dixon's statistic}

Likes ${ }^{16}$ proposed Dixon's ${ }^{15}$ statistic to identify suspected observations in exponential samples. This is given by

$$
Z=\frac{X_{(s)}-X_{(r)}}{X_{(q)}-X_{(p)}}, 1 \leq p \leq r<s \leq q \leq n ; q-p>s-r
$$

The distribution of $Z$ is shown to be

$$
\begin{aligned}
& 1-F_{Z}(z) \\
= & \frac{(n-p) !}{(n-q) !}(1-z)\left\{\sum_{i=1}^{q-s} \sum_{k=1}^{s-r}\left[\frac{(-1)^{i+k}(q-r-i) ![(n-s+k) z+(n-q+i)(1-z)]^{-1}}{(i-1) !(k-1) !(q-s-i) !(s-r-k) !(q-p-i) !(n-s+k)}\right]\right. \\
& \left.+\sum_{j=1}^{r-p} \sum_{k=1}^{s-r}\left[\frac{(-1)^{q-s+j+k}(s-r+j-1) ![(n-s+k) z+(n-r+k)(1-z)]^{-1}}{(j-1) !(k-1) !(r-p-j) !(s-r-k) !(q-r+j-1) !(n-s+k)}\right]\right\}
\end{aligned}
$$

\section{References}

1. Chakraborti S, Human SW, Graham MA. Phase I statistical process control charts: An overview and some results. Quality Engineering 2009; 21:52-62.

2. Montegomery DC. Introduction to Statistical Quality Control (6th edition). Wiley: New York, 2009.

3. Lucas JM. Counted data CUSUMs. Technometrics 1985; 27:129-144.

4. Vardeman S, Ray D. Average run lengths for CUSUM schemes when observations are exponentially distributed. Technometrics 1985; 27:145-150. 
5. Gan FF. Designs of one- and two-sided exponential EWMA charts. Journal of Quality Technology 1998; 30:55-69.

6. Liu JY, Xie M, Goh TN, Sharma PR. A comparative study of exponential time between events charts. Quality Technology and Quantitative Management 2006; 3:347-359.

7. Nelson LS. A control chart for parts-per-million nonconforming items. Journal of Quality Technology 1994; 26:239-240.

8. Kittlitz RG. Transforming the exponential for SPC applications. Journal of Quality Technology 1999; 31:301-308.

9. Kao S. Normalization of the origin-shifted exponential distribution for control chart construction. Journal of Applied Statistics 2010; 27:1067-1087.

10. Jones LA, Champ CW. Phase I control charts for times between events. Quality and Reliability Engineering International 2002; 18:479-488.

11. Pehlivan C, Testik MC. Impact of model misspecification on the exponential EWMA charts: A robustness Study when the time-between-events are not exponential. Quality and Reliability Engineering International 2010; 26:177-190.

12. Borror CM, Keats JB, Montgomery DC. Robustness of the time between events CUSUM. International Journal of Production Research 2003; 41:3435-3444.

13. Dovoedo YH, Chakraborti S. Boxplot-based Phase I Control Charts for Time Between Events. Quality and Reliability Engineering International 2012; 28:123130.

14. Dovoedo YH, Chakraborti S. On some properties of a simple and more general boxplot-type method for identifying outliers. American Statistical Association Proceedings of the Joint Statistical Meetings, Alexandria, VA, 2009; 3900-3908.

15. Dixon WJ. Processing data for outliers. Biometrics 1953; 9:74-89.

16. Likes J. Distribution of Dixon's statistics in the case of an exponential population. Metrika 1966; 11:46-54. 


\section{Authors' biographies}

N. Kumar is an Assistant Professor in the Department of Statistics, M.G. Kashi Vidyapith, Varanasi, India. He is currently a Postdoctoral Fellow at the Department of Statistics in the University of Pretoria, Pretoria, RSA. He received his Master and D. Phil. Degree in Statistics from the University of Allahabad, Allahabad, India. His research interests include statistical outlier detection, statistical process control and panel data models.

S. Chakraborti is a Professor of Statistics in the University of Alabama in Tuscaloosa, Alabama, U.S.A. His research interests include theory and applications of nonparametric and robust statistical methodologies with emphasis on statistical process control. Professor Chakraborti is a Fellow of the American Statistical Association and an elected member of the International Statistical Institute. He is the author or the co-author of over 80 publications including the book Nonparametric Statistical Inference published by Taylor and Francis 
Table 1: Fence constants for the proposed one-sided control chart

\begin{tabular}{crrrrr}
\hline & \multicolumn{5}{c}{ Number of Phase I observations $n$} \\
\cline { 2 - 6 }$\alpha_{0}$ & 10 & 15 & 20 & 25 & 30 \\
\hline 0.01 & 280.0482 & 620.906 & 823.4736 & 1104.8554 & 1308.5535 \\
0.05 & 54.4843 & 121.2247 & 160.9200 & 216.0615 & 255.9534 \\
0.1 & 26.2788 & 58.74 & 78.0677 & 104.9175 & 124.3375 \\
0.2 & 12.1576 & 27.4521 & 36.5802 & 49.2624 & 58.4318 \\
\hline
\end{tabular}

Table 2: Fence constants for the proposed two-sided control chart

\begin{tabular}{|c|c|c|c|c|c|c|c|c|c|c|}
\hline \multirow[b]{3}{*}{$\alpha_{0}$} & \multicolumn{10}{|c|}{ Number of Phase I observations $n$} \\
\hline & \multicolumn{2}{|c|}{10} & \multicolumn{2}{|c|}{15} & \multicolumn{2}{|c|}{20} & \multicolumn{2}{|c|}{25} & \multicolumn{2}{|c|}{30} \\
\hline & $k_{1}$ & $k_{2}$ & $k_{1}$ & $k_{2}$ & $k_{1}$ & $k_{2}$ & $k_{1}$ & $k_{2}$ & $k_{1}$ & $k_{2}$ \\
\hline$\overline{0.01}$ & 560.1742 & 1161.1827 & 1240.2384 & 1859.9880 & 1644.3525 & 2708.1128 & 2205.6963 & 4112.5772 & 2611.8057 & 5069.8775 \\
\hline 0.05 & 109.0588 & 225.1749 & 240.9067 & 360.5456 & 319.2869 & 524.9170 & 428.1643 & 796.9483 & 506.9276 & 982.5032 \\
\hline 0.1 & 52.6642 & 108.1645 & 115.9775 & 173.0985 & 153.6365 & 251.9917 & 205.9495 & 382.4547 & 243.7901 & 471.5313 \\
\hline 0.2 & 24.4568 & 49.64082 & 53.4879 & 79.3422 & 70.7777 & 115.4788 & 94.7966 & 175.1298 & 112.1674 & 215.9476 \\
\hline
\end{tabular}

Table 3: Empirical overall false alarm rates for the one-sided control charts

\begin{tabular}{rlllll}
\multirow{2}{*}{$\alpha_{0}$} & & \multicolumn{4}{c}{ Distribution } \\
\cline { 3 - 6 } & Method & Gamma(1.2,1) & Gamma(1.1,1) & Gamma(0.9,1) & Gamma $(0.8,1)$ \\
\hline 0.01 & Proposed & $0.00921(7.9 \%)$ & $0.00954(4.6 \%)$ & $0.00971(2.9 \%)$ & $0.01000(0.0 \%)$ \\
& DC & $0.01508(50.8 \%)$ & $0.01148(14.8 \%)$ & $0.00789(21.1 \%)$ & $0.00605(39.5 \%)$ \\
& JC & $0.00256(74.4 \%)$ & $0.00507(49.3 \%)$ & $0.01965(96.5 \%)$ & $0.04009(300.9 \%)$ \\
0.05 & Proposed & $0.05033(0.7 \%)$ & $0.05046(0.9 \%)$ & $0.04977(0.5 \%)$ & $0.04992(0.2 \%)$ \\
& DC & $0.06864(37.3 \%)$ & $0.05781(15.6 \%)$ & $0.04182(16.4 \%)$ & $0.03238(35.2 \%)$ \\
& JC & $0.01747(65.1 \%)$ & $0.02920(41.6 \%)$ & $0.08521(70.4 \%)$ & $0.14140(182.8 \%)$ \\
& Proposed & $0.10035(0.3 \%)$ & $0.10062(0.6 \%)$ & $0.09980(0.2 \%)$ & $0.10044(0.4 \%)$ \\
& DC & $0.13323(33.2 \%)$ & $0.11625(16.3 \%)$ & $0.08442(15.6 \%)$ & $0.06565(34.4 \%)$ \\
& JC & $0.04075(59.3 \%)$ & $0.06426(35.7 \%)$ & $0.15469(54.7 \%)$ & $0.23709(137.1 \%)$ \\
& Proposed & $0.20070(0.3 \%)$ & $0.20178(0.9 \%)$ & $0.20088(0.4 \%)$ & $0.20185(0.9 \%)$ \\
& DC & $0.25265(26.3 \%)$ & $0.22573(12.9 \%)$ & $0.17322(13.4 \%)$ & $0.14038(29.8 \%)$ \\
& JC & $0.09845(50.8 \%)$ & $0.13859(30.7 \%)$ & $0.28209(41.0 \%)$ & $0.39207(96.0 \%)$ \\
\hline
\end{tabular}


Table 4: Empirical overall false alarm rates for the two-sided control charts

\begin{tabular}{|c|c|c|c|c|c|}
\hline \multirow[b]{2}{*}{$\alpha_{0}$} & \multirow[b]{2}{*}{ Method } & \multicolumn{4}{|c|}{ Distribution } \\
\hline & & $\operatorname{Gamma}(1.2,1)$ & $\operatorname{Gamma}(1.1,1)$ & $\operatorname{Gamma}(0.9,1)$ & $\operatorname{Gamma}(0.8,1)$ \\
\hline \multirow[t]{3}{*}{$\overline{0.01}$} & Proposed & $0.01006(0.6 \%)$ & $0.00987(1.3 \%)$ & $0.01005(0.5 \%)$ & $0.01000(0.0 \%)$ \\
\hline & $\mathrm{DC}$ & $0.01135(13.5 \%)$ & $0.01044(4.4 \%)$ & $0.00972(2.8 \%)$ & $0.01028(2.8 \%)$ \\
\hline & $\mathrm{JC}$ & $0.00281(71.9 \%)$ & $0.00494(50.6 \%)$ & $0.01983(98.3 \%)$ & $0.03941(294.1 \%)$ \\
\hline \multirow[t]{3}{*}{0.05} & Proposed & $0.04941(1.2 \%)$ & $0.05056(1.1 \%)$ & $0.05026(0.5 \%)$ & $0.04996(0.1 \%)$ \\
\hline & $\mathrm{DC}$ & $0.05536(10.7 \%)$ & $0.05149(3.0 \%)$ & $0.04833(3.3 \%)$ & $0.04856(2.9 \%)$ \\
\hline & $\mathrm{JC}$ & $0.01791(64.2 \%)$ & $0.03000(40.0 \%)$ & $0.08027(60.5 \%)$ & $0.13365(167.3 \%)$ \\
\hline \multirow[t]{3}{*}{0.1} & Proposed & $0.09923(0.8 \%)$ & $0.10091(0.9 \%)$ & $0.10159(1.6 \%)$ & $0.101841 .8 \%)$ \\
\hline & $\mathrm{DC}$ & $0.10860(8.6 \%)$ & $0.10354(3.5 \%)$ & $0.09506(4.9 \%)$ & $0.09673(3.3 \%)$ \\
\hline & $\mathrm{JC}$ & $0.04125(58.8 \%)$ & $0.06263(37.4 \%)$ & $0.14634(46.3 \%)$ & $0.22658(126.6 \%)$ \\
\hline \multirow[t]{3}{*}{0.2} & Proposed & $0.20310(1.6 \%)$ & $0.20010(0.0 \%)$ & $0.20500(2.5 \%)$ & $0.20130(0.6 \%)$ \\
\hline & $\mathrm{DC}$ & $0.22140(10.7 \%)$ & $0.21020(5.1 \%)$ & $0.19180(4.1 \%)$ & $0.18630(6.9 \%)$ \\
\hline & $\mathrm{JC}$ & $0.09380(53.1 \%)$ & $0.12780(36.1 \%)$ & $0.25660(28.3 \%)$ & $0.36430(82.2 \%)$ \\
\hline
\end{tabular}


Table 5: OOC performance of the one-sided Phase I control charts

\begin{tabular}{|c|c|c|c|c|c|c|}
\hline \multirow[b]{2}{*}{$\alpha_{0}$} & \multirow[b]{2}{*}{$\mathrm{t}$} & \multirow[b]{2}{*}{ Method } & \multicolumn{4}{|c|}{ Shift size $\delta$} \\
\hline & & & -0.05 & -0.1 & -0.25 & -0.5 \\
\hline \multirow[t]{9}{*}{$\overline{0.01}$} & $\mathrm{t}=1$ & Proposed & 0.01008 & 0.00961 & 0.01007 & 0.00977 \\
\hline & & $\mathrm{DC}$ & 0.01017 & 0.01030 & 0.00985 & 0.00956 \\
\hline & & $\mathrm{JC}$ & 0.01012 & 0.00984 & 0.01033 & 0.00989 \\
\hline & $t=3$ & Proposed & 0.01015 & 0.00993 & 0.00993 & 0.00966 \\
\hline & & $\mathrm{DC}$ & 0.00953 & 0.01000 & 0.01004 & 0.00953 \\
\hline & & $\mathrm{JC}$ & 0.01037 & 0.01015 & 0.01016 & 0.01054 \\
\hline & $\mathrm{t}=5$ & Proposed & 0.01017 & 0.01017 & 0.00965 & 0.01016 \\
\hline & & $\mathrm{DC}$ & 0.00966 & 0.01005 & 0.01000 & 0.00987 \\
\hline & & $\mathrm{JC}$ & 0.01023 & 0.01043 & 0.01059 & 0.01013 \\
\hline \multirow[t]{9}{*}{0.05} & $\mathrm{t}=1$ & Proposed & 0.04970 & 0.05025 & 0.04946 & 0.05041 \\
\hline & & $\mathrm{DC}$ & 0.05010 & 0.05006 & 0.04960 & 0.04833 \\
\hline & & $\mathrm{JC}$ & 0.04977 & 0.05105 & 0.04939 & 0.05085 \\
\hline & $t=3$ & Proposed & 0.05024 & 0.04973 & 0.05022 & 0.05009 \\
\hline & & $\mathrm{DC}$ & 0.05107 & 0.05055 & 0.04890 & 0.04822 \\
\hline & & $\mathrm{JC}$ & 0.04968 & 0.04899 & 0.05093 & 0.05139 \\
\hline & $\mathrm{t}=5$ & Proposed & 0.05176 & 0.05063 & 0.05095 & 0.05118 \\
\hline & & $\mathrm{DC}$ & 0.04891 & 0.05025 & 0.04999 & 0.04736 \\
\hline & & $\mathrm{JC}$ & 0.04955 & 0.05052 & 0.04985 & 0.05210 \\
\hline \multirow[t]{9}{*}{0.1} & $\mathrm{t}=1$ & Proposed & 0.09956 & 0.10009 & 0.09958 & 0.10013 \\
\hline & & $\mathrm{DC}$ & 0.10029 & 0.10039 & 0.09992 & 0.09616 \\
\hline & & $\mathrm{JC}$ & 0.10152 & 0.10118 & 0.10070 & 0.10314 \\
\hline & $\mathrm{t}=3$ & Proposed & 0.09825 & 0.09864 & 0.09985 & 0.09961 \\
\hline & & $\mathrm{DC}$ & 0.10157 & 0.10019 & 0.09929 & 0.09679 \\
\hline & & $\mathrm{JC}$ & 0.09910 & 0.09908 & 0.10348 & 0.10474 \\
\hline & $\mathrm{t}=5$ & Proposed & 0.10018 & 0.09998 & 0.09892 & 0.10116 \\
\hline & & $\mathrm{DC}$ & 0.09954 & 0.09839 & 0.09842 & 0.09715 \\
\hline & & $\mathrm{JC}$ & 0.10044 & 0.09951 & 0.10072 & 0.10156 \\
\hline \multirow[t]{9}{*}{0.2} & $\mathrm{t}=1$ & Proposed & 0.19973 & 0.20241 & 0.19704 & 0.19838 \\
\hline & & $\mathrm{DC}$ & 0.19833 & 0.19913 & 0.19781 & 0.19584 \\
\hline & & $\mathrm{JC}$ & 0.20093 & 0.19979 & 0.20086 & 0.20411 \\
\hline & $\mathrm{t}=3$ & Proposed & 0.19839 & 0.19961 & 0.20018 & 0.20828 \\
\hline & & $\mathrm{DC}$ & 0.20158 & 0.20180 & 0.19968 & 0.18724 \\
\hline & & $\mathrm{JC}$ & 0.19873 & 0.20096 & 0.20233 & 0.21122 \\
\hline & $\mathrm{t}=5$ & Proposed & 0.19940 & 0.20078 & 0.19984 & 0.20177 \\
\hline & & $\mathrm{DC}$ & 0.19959 & 0.20182 & 0.19670 & 0.19677 \\
\hline & & $\mathrm{JC}$ & 0.19848 & 0.19717 & 0.20466 & 0.20701 \\
\hline
\end{tabular}


Table 6: OOC performance of the two-sided Phase I control charts

\begin{tabular}{|c|c|c|c|c|c|c|}
\hline \multirow[b]{2}{*}{$\alpha_{0}$} & \multirow[b]{2}{*}{$t$} & \multirow[b]{2}{*}{ Method } & \multicolumn{4}{|c|}{ Shift size $\delta$} \\
\hline & & & -0.05 & -0.1 & -0.25 & -0.5 \\
\hline \multirow[t]{9}{*}{$\overline{0.01}$} & $\mathrm{t}=1$ & Proposed & 0.00990 & 0.00996 & 0.01002 & 0.00997 \\
\hline & & $\mathrm{DC}$ & 0.00987 & 0.00993 & 0.00991 & 0.01012 \\
\hline & & $\mathrm{JC}$ & 0.00989 & 0.00974 & 0.01067 & 0.01082 \\
\hline & $\mathrm{t}=3$ & Proposed & 0.00958 & 0.01042 & 0.00997 & 0.01000 \\
\hline & & DC & 0.01024 & 0.00983 & 0.01031 & 0.01115 \\
\hline & & $\mathrm{JC}$ & 0.01041 & 0.00996 & 0.01060 & 0.01318 \\
\hline & $\mathrm{t}=5$ & Proposed & 0.01009 & 0.00953 & 0.01008 & 0.01082 \\
\hline & & $\mathrm{DC}$ & 0.00971 & 0.01006 & 0.01042 & 0.01132 \\
\hline & & $\mathrm{JC}$ & 0.01007 & 0.01058 & 0.01116 & 0.01545 \\
\hline \multirow[t]{9}{*}{0.05} & $\mathrm{t}=1$ & Proposed & 0.04899 & 0.05058 & 0.04955 & 0.05101 \\
\hline & & $\mathrm{DC}$ & 0.05094 & 0.04890 & 0.05098 & 0.05037 \\
\hline & & $\mathrm{JC}$ & 0.04891 & 0.04897 & 0.04960 & 0.05304 \\
\hline & $\mathrm{t}=3$ & Proposed & 0.04987 & 0.05020 & 0.05033 & 0.05041 \\
\hline & & $\mathrm{DC}$ & 0.04957 & 0.04920 & 0.05197 & 0.05188 \\
\hline & & $\mathrm{JC}$ & 0.04821 & 0.04952 & 0.05155 & 0.05913 \\
\hline & $\mathrm{t}=5$ & Proposed & 0.04832 & 0.05026 & 0.04904 & 0.05072 \\
\hline & & $\mathrm{DC}$ & 0.05037 & 0.04999 & 0.05192 & 0.05526 \\
\hline & & $\mathrm{JC}$ & 0.04983 & 0.04834 & 0.05284 & 0.06777 \\
\hline \multirow[t]{9}{*}{0.1} & $\mathrm{t}=1$ & Proposed & 0.10052 & 0.09872 & 0.10064 & 0.09993 \\
\hline & & DC & 0.10025 & 0.09879 & 0.10212 & 0.10125 \\
\hline & & $\mathrm{JC}$ & 0.09657 & 0.09519 & 0.09704 & 0.10128 \\
\hline & $\mathrm{t}=3$ & Proposed & 0.10056 & 0.10060 & 0.10285 & 0.10048 \\
\hline & & $\mathrm{DC}$ & 0.10086 & 0.10012 & 0.10277 & 0.10279 \\
\hline & & $\mathrm{JC}$ & 0.09686 & 0.09723 & 0.10227 & 0.11356 \\
\hline & $\mathrm{t}=5$ & Proposed & 0.09756 & 0.09938 & 0.10201 & 0.10175 \\
\hline & & $\mathrm{DC}$ & 0.09983 & 0.10227 & 0.10215 & 0.11005 \\
\hline & & $\mathrm{JC}$ & 0.09585 & 0.09647 & 0.10146 & 0.12673 \\
\hline \multirow[t]{9}{*}{0.2} & $\mathrm{t}=1$ & Proposed & 0.20019 & 0.19734 & 0.19894 & 0.20263 \\
\hline & & $\mathrm{DC}$ & 0.19984 & 0.19918 & 0.20085 & 0.20116 \\
\hline & & $\mathrm{JC}$ & 0.18586 & 0.18337 & 0.18427 & 0.19393 \\
\hline & $\mathrm{t}=3$ & Proposed & 0.20008 & 0.20120 & 0.19995 & 0.20280 \\
\hline & & $\mathrm{DC}$ & 0.19909 & 0.20120 & 0.20363 & 0.20335 \\
\hline & & $\mathrm{JC}$ & 0.18372 & 0.18439 & 0.18715 & 0.21261 \\
\hline & $\mathrm{t}=5$ & Proposed & 0.20063 & 0.20112 & 0.20119 & 0.20290 \\
\hline & & $\mathrm{DC}$ & 0.20074 & 0.20108 & 0.20623 & 0.21133 \\
\hline & & $\mathrm{JC}$ & 0.18366 & 0.18414 & 0.19366 & 0.23005 \\
\hline
\end{tabular}

Table 7: Time between failures data

\begin{tabular}{|c|c|c|c|c|c|c|c|c|c|}
\hline 1.24 & 6.69 & 9.77 & 1.23 & 14.03 & 18.07 & 3.90 & 13.61 & 18.47 & 12.85 \\
\hline 52.32 & 14.75 & 4.69 & 0.18 & 13.61 & 4.57 & 0.28 & 7.08 & 12.00 & 5.15 \\
\hline 6.09 & 20.41 & 5.93 & 19.03 & 13.65 & 6.37 & 2.06 & 3.30 & 6.91 & 12.08 \\
\hline
\end{tabular}

